\title{
Fuzzy Ambient Intelligence for Intelligent Office Environments
}

\author{
Saifullizam Puteh, Caroline Langensiepen and Ahmad Lotfi \\ School of Science and Technology \\ Nottingham Trent University, Nottingham, United Kingdom \\ Email: \{saifullizam.puteh, caroline.langensiepen, ahmad.lotfi\}@ntu.ac.uk
}

\begin{abstract}
In this paper we present an ambient intelligence system for modelling and control of power use within an office environment. We define a multi-scale model of the office worker, consisting of a coarse grained office user profile, together with a fine grained characteristics model to summarise their behaviours. Sensor data gathered from individual offices are used to create these models. Collected data are fuzzified to produce meaningful and understandable descriptions of the worker's daily activities. These models of the individuals form the basis of a Dynamic Power Usage Scheme (DPUS) to control individual offices. A trial version of the system has been implemented using individual academic staff offices in a university campus. Experiments have shown that even a simplistic user profile can lead to significant power savings from appropriate control of computers in an office environment.
\end{abstract}

Index Terms-Ambient Intelligence, Fuzzy Control, Fuzzy Systems, Intelligent Environments, Energy Saving, Energy Management

\section{INTRODUCTION}

In modern office environments, personal computers (PC), lighting systems and heating/cooling system are the main energy consumers. Many companies would like to reduce their energy usage for two reasons. The first is that they worry about the environment and want to reduce the impact they have on it. The second reason, and most likely the one that companies care about most, is cost. For example, PCs waste a lot of energy due to being left on for long periods of time when not in use. Even though they have power management modes to reduce their energy consumptions when they are not in use, these are not always used. Some workplaces incorporate reactive systems such as passive infra-red (PIR) activated lighting, but these can be activated/deactivated inappropriately. Heating systems often work on the assumption of a 9:00am to 5:00pm presence, five days a week, whereas an individual office worker may have a different schedule, including long periods out of the office.

The aim of the our research is to incorporate a Dynamic Power Usage Scheme (DPUS) in an office environment to control PCs, lighting and temperature based on individual office users. To achieve this, it is important to understand each user's profile initially before adapting the environment to the users requirements. For example, instead of waiting half an hour for a timer to turn off the PC, the system could detect that the user is no longer in the room and the computer is not performing any work. This would cause the DPUS to then put the PC into an energy saving mode.
The main focus of this paper is to create a fuzzy characteristics matrix to summarise the activities of an office user based on data collected from a sensor network. The profile matrix is presented as fuzzy values which indicate the likelihood of the sequence of activities in future.

This paper is organised as follows: in Section II of this paper a short summary of the related work regarding Ambient Intelligence (AmI) in office environments is presented. In Section III proposed system architecture is explained followed by the pre-processing data stage explained in Section IV. Section VI explain the proposed technique for generating fuzzy user profiles. Details of our experiments are presented in Section VII. Section VIII draws some conclusions and discusses the direction in which the research needs to progress.

\section{RELATED WORK}

Jori Reijula et-al. [1] defined the concept of an intelligent work environment and evaluated its meaning from a user's perspective. They take a multi-professional approach to foresee the possibilities and challenges awaiting future users of intelligent working environments, including the importance of ergonomics when designing intelligent work environments of the future.

The "intelligent and personalized-energy conservation system (iPower)", developed by Ye et-al. [2] is a good example of how a smart environment can help to reduce power consumption in an office. Sensor nodes placed around the environment are able to monitor different aspects of the office working environment. When these different sensor measurements are combined, a picture of the environment can be processed which allows for sensible decisions to be made on how to control that environment. Using wireless sensors is appropriate as it allows them to be placed in good locations very easily. The concept of smart furniture is also introduced by the example of using a pressure plate on a chair to indicate if someone is sitting on it. This could be used to determine if a user is at their desk, and so could be using their PC.

A sensor-based modelling and prediction of user behaviour in intelligent buildings is proposed in [3]. The proposed system connects behavioural patterns to a building energy and comfort management system, although most of the work is based on the simulation tools. Some researchers have investigated the comfort of the worker in work place. Haynes [5] points out that while there is sufficient evidence to support claims that 
office comfort affects productivity, there is no agreement as to how office comfort should be measured. In [4], authors used multiple survey items to assess worker discomfort, sleep quality and hindrance, in order to relate building aspects to any physical and/or psychological effects.

Application of fuzzy data analysis in AmI have already been investigated in telecare systems [6] [7]. Due to the high degree of uncertainty, it is shown that fuzzy AmI would be able to answer high level queries such as "is the person eating regularly". Applications of adaptive fuzzy control system in smart home are reported in [8]. The research has attempted to propose an adaptive home to adapt to its inhabitants' living style. Similar applications using embedded agents are reported in [9] [10].

A comprehensive review of literature and related work in this area are presented in [11] with details in three categories, namely; modelling the office or building, human behaviour modelling and comfort assessments.

\section{Proposed System Architecture}

A schematic diagram of the proposed DPUS is illustrated in Fig. 1. This proposed prototype system has four parts including a wirless sensor network installed in each office, a PC monitorig application, a database where all activities are logged and a control server application to control the environment. More details are given below.

\section{A. Wireless Sensor Network Data Collection System}

Wireless sensor monitoring is employed to collect office user behavioural information. In total, each office is equipped with 9 sensors measuring different activities and properties of the testbed environment. The sensors include:

- Motion sensor (On/Off)

- Door entry point sensor (On/Off)

- Windows entry point sensor (On/Off)

- Pressure sensor measuring chair occupancy (On/Off)

- Room temperature

- Room humidity

- Outside temperature

- Room light intensity

- Ambient light intensity

The sensor network provides information regarding the occupancy and ambient conditions. Occupancy detection is provided by PIR motion sensors and door entry switches. PIR motion sensors are low cost and readily available, though their typical activity thresholds mean that a worker quietly reading or writing a document would not cause them to signal. Door sensors are relatively unambiguous. However, a visitor to an office would also trigger the open-shut sequence, so the office occupancy could not necessarily be deduced simply from the door. Thus occupancy needs to be deduced from a combination of these signals together with additional information provided by other sensors.

The ambient conditions are recorded via temperature, humidity and light sensors (inside the office and external to the building). The response of the office worker can also be

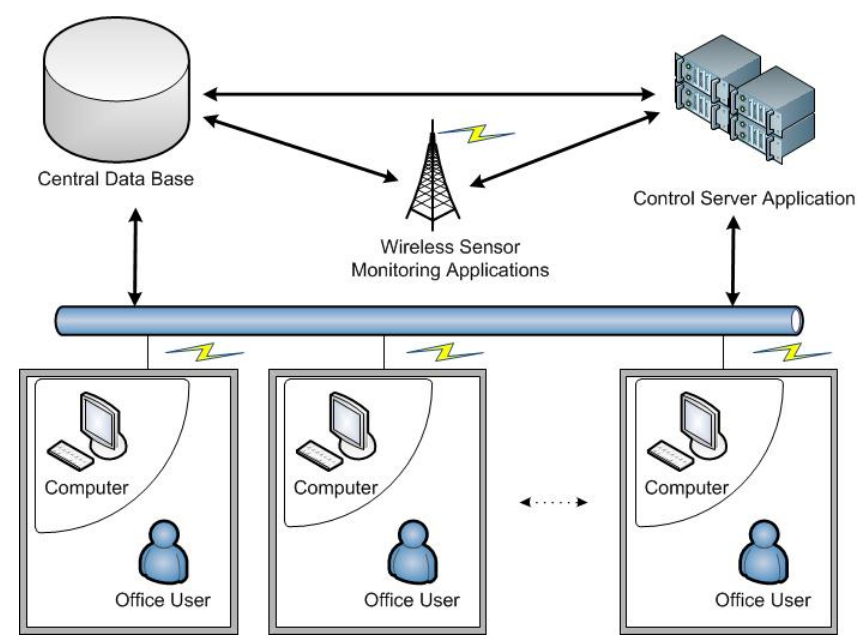

Fig. 1: Proposed System Architecture for Dynamic Power Usage Scheme.

captured via switches on the windows (as for doors) to detect when they have been opened or shut.

\section{B. Monitoring Application}

Information regarding the office PC is collected using a monitoring application agent installed on the PC to be monitored. A simple monitoring application logs keyboard and mouse activities and this information is recorded in the database along with the data collected from the wireless sensor network. A monitoring application software agent will send the information directly to the database using the local area network.

\section{Database}

A database records events triggered by the $\mathrm{PC}$ and wireless sensors and also stores the state of the PC and wireless sensors. Table I shows a sample of the database format. The database logs individual events in order for any further analysis.

\section{Control Server Application}

The control server application is responsible for analysing the state of the environment and then deciding whether it is appropriate to activate the $\mathrm{PC}$, lighting or heating in that environments' power management mode. To do this it needs to be able to receive messages from the wireless sensor

TABLE I: Sample of database data format.

\begin{tabular}{||l|l|c|c|c||}
\hline Data & Time & Sensor ID & Status & Room \\
\hline $1 / 12 / 2011$ & $12: 20: 01$ & 1 & 1 & 2 \\
\hline $1 / 12 / 2011$ & $12: 20: 03$ & 2 & 1 & 2 \\
\hline $1 / 12 / 2011$ & $12: 21: 05$ & 3 & 0 & 3 \\
\hline $1 / 12 / 2011$ & $12: 21: 08$ & 2 & 0 & 3 \\
\hline $1 / 12 / 2011$ & $12: 28: 05$ & 2 & 1 & 3 \\
\hline $1 / 12 / 2011$ & $12: 28: 05$ & 2 & 1 & 1 \\
\hline $1 / 12 / 2011$ & $12: 29: 01$ & 2 & 0 & 3 \\
\hline
\end{tabular}




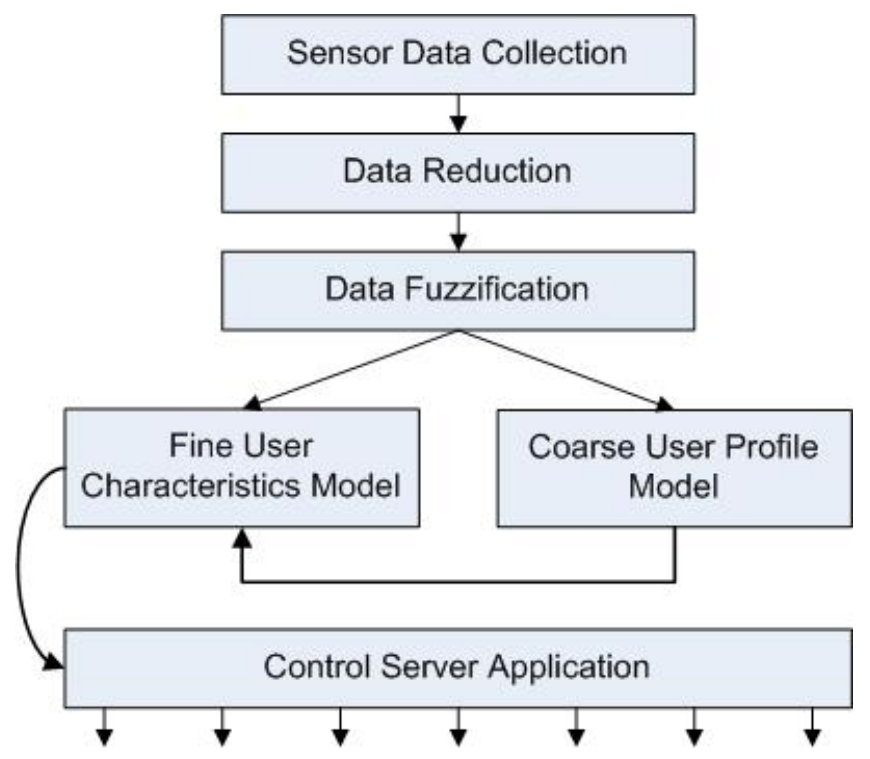

Fig. 2: Control Server Application.

monitoring application. A client-server architecture will be used between the PC monitoring application, the control server application and the wireless sensor monitoring application. This is for two reasons. The first is that the PC monitoring application and the wireless sensor monitoring application need to communicate any changes in the environment's and PC's state to the server. The second is that the control server application needs to issue messages to the PC monitoring application to activate its power management and broadcast messages across the network to wake up PCs and also activate lighting as well as heating.

In order for the control server application to carry out its role, the workers patterns have to be analysed. To achieve this, the raw sensor data in the database has to be transformed into meaningful information about aspects of the worker. The process of distilling the information is shown in Fig. 2. The first stage is to pre-process the raw data to make it more compact and efficient. To remove irrelevant details, we then fuzzify the data into meaningful categories. This allows us to use two stages of characterisation and profiling the worker. Initially a coarse grain model of the worker's activities is constructed to form their profile. This allows a simple rulebased control scheme for power management. Subsequently, the detailed information is used to form a fine grain model of the characteristics, in order to improver the quality of control produced from the coarse profile. The following sections give more details of the stages involved.

\section{Data Pre-Processing}

Collected data from each office represent the user profile for that environment. Different users have different preferences and it is essential to summarise the information into meaningful knowledge within the control server application to control each office environment effectively. Two stages of pre-processing are implemented.

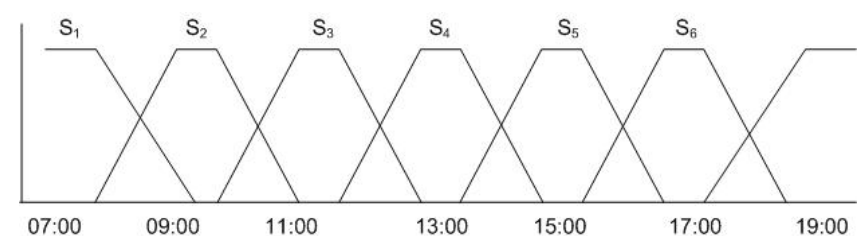

(a) Start Time (hour)

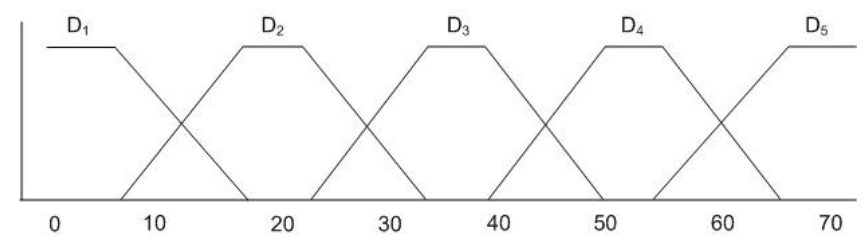

(b) Duration (minutes)

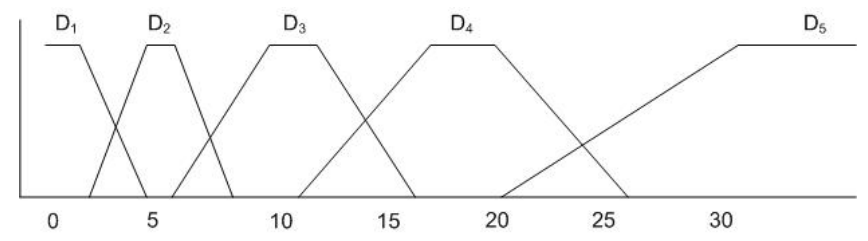

(c) Duration (minutes)

Fig. 3: Fuzzy partitions (a) start time for all events (b) duration for chair occupancy (c) duration for PC activities.

\section{A. Data Reduction}

From our earlier research [12], it is found that converting the data into a start-time, $S T_{k}(t)$, and duration, $D U_{k}(t)$, sequences would be the most efficient way of presenting the data for any further processing. Converting the raw data into $S T_{k}(t)$ and $D U_{k}(t)$ for each activity, $k$, will summarise the data and present them in a compact format. Table II shows a sample of data in start-time and duration format for binary signals.

\section{B. Data Fuzzification}

Knowing the exact time (in seconds or even minutes) for the start-time and duration of an activity would not be very significant. For example if an office worker is usually come to his/her office early morning, then this information should be enough to create a user profile without specifying the exact time every day. Therefore, in order to manipulate categorical data rather than numerical data, $S T_{k}(t)$ and $D U_{k}(t)$ for all sensor readings are replaced by their membership in several fuzzy sets.

TABLE II: A sample of data in start-time and duration format for binary signals.

\begin{tabular}{||l|c|c|c||}
\hline Data & Sensor ID $(\mathrm{k})$ & $S T_{k}(t)$ & $D U_{k}(t)$ \\
\hline $1 / 12 / 2011$ & 1 & $12: 20: 12$ & 29 \\
\hline $1 / 12 / 2011$ & 2 & $12: 22: 25$ & 22 \\
\hline $1 / 12 / 2011$ & 3 & $12: 22: 28$ & 20 \\
\hline $1 / 12 / 2011$ & 2 & $14: 12: 02$ & 45 \\
\hline
\end{tabular}




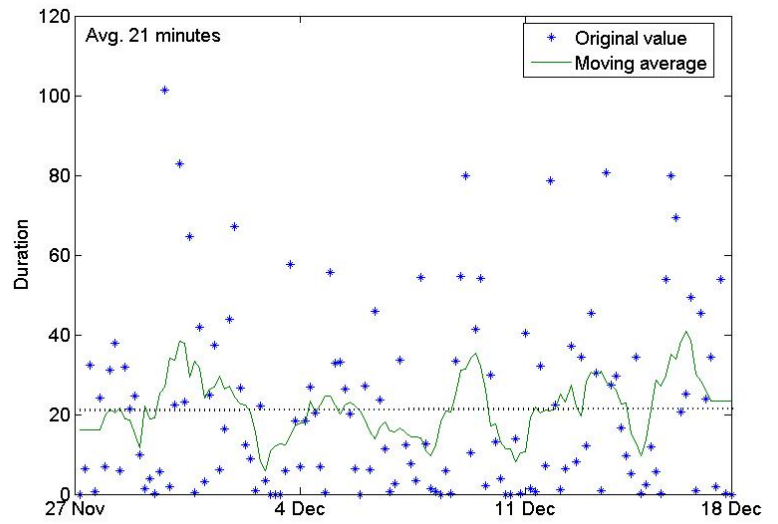

Fig. 4: A sample of chair occupancy duration over three weeks.

Time of the day is split into $m=7$ overlapping fuzzy sets, $\tilde{S}_{i}, i=1, \ldots, m$. They are $\tilde{S}_{1}$ : EarlyMorning, $\tilde{S}_{2}$ : Morning, $\tilde{S}_{3}$ : LateMorning, $\tilde{S}_{4}$ : Noon, $\tilde{S}_{5}$ : EarlyAfternoon, $\tilde{S}_{6}$ : Afternoon, $\tilde{S}_{7}$ : LateAfternoon. Fuzzy partition for $S T_{k}(t)$ is shown in Fig. 3-a.

Duration of events is fuzzified on an event basis. Working in front of a PC for a "short time" is not the same as opening the window for a "short time". Fig. 3-b and Fig. 3c illustrate fuzzy values for duration of chair occupancy and PC activities respectively. For all events, duration is split into $n=5$ overlapping fuzzy sets, $\tilde{D}_{j}, j=1, \ldots, n$. They are $\tilde{D}_{1}$ : VeryShort, $\tilde{D}_{2}$ : Short, $\tilde{D}_{3}$ : Medium, $\tilde{D}_{4}$ : Long, $\tilde{D}_{5}$ : VeryLong.

The degree of membership, $\mu$ (which is the degree to which the event satisfies the $S T_{k}(t)$ and $D U_{k}(t)$ fuzzy labels) are calculated and these grades will be used to create a profile for a user.

\section{WORKER PROFILE}

Each office user's working attitude can be categorised into very conservative clusters. This can be separated into categories for starting the day. A user can be:

- $A_{1}$ - Early starter

- $A_{2}$ - Normal starter (around 9:00am)

- $A_{3}$ - Late starter

and more or less the same will apply for finishing the day. They can be categorised as:

- $B_{1}$ - Early end

- $B_{2}$ - Normal end (around 5:00pm)

- $B_{3}$ - Late end

Depending on the nature of the office computer work, an office user can be classified as:

- $C_{1}$ - Light computer user

- $C_{2}$ - Medium computer user

- $C_{3}$ - Heavy computer user

and finally, the behaviour of a person can be classified as:

- $H_{1}$ - Very routine habits

- $\mathrm{H}_{2}$ - Routine habits

- $H_{3}$ - Random habits
Each person will be clustered into one of the 81 fuzzy clusters. For example, a user could be considered as:

\#1: $\quad$ starts the day $A_{1}$ and finishes the day $B_{2}$, their computer usage is $C_{3}$ and overall this person's work pattern is $\mathrm{H}_{2}$.

\#2: $\quad$ starts the day $A_{2}$ and finishes the day $B_{1}$, their computer usage is $C_{1}$ and overall this person's work pattern is $\mathrm{H}_{3}$.

Histograms of the data for room occupancy and door activation will suggest the person's work start and end. The third category is identified from the PC activities, and their work pattern is identified from the total number of changes of activity during a day. For example 50 and over changes will identity a person as $H_{3}$. Therefore, knowing that a person has a random habit, it is not necessary to turn on/off the computer and room light so frequently.

These patterns are not fixed and they may vary on different days of a week depending on the nature of the work. For example, Fig. 4 shows a sample of chair occupancy duration over three weeks for a worker. The solid line shows the daily moving average for the same dataset. The worker clearly has different patterns on different days of the week. The coarse profile, therefore, considers only the average in order to provide the first stage of modelling. There will be some seasonal changes so that the pattern of work may very during the summer and winter time. The research intends to identify the pattern of energy usage on a daily and weekly basis only. Further investigations are required to study this on a monthly or yearly basis.

\section{FUZZY USERS CHARACTERISTICS}

Start time and duration of each event are represented in membership values for all fuzzy labels. Therefore for each event, two arrays of membership values for $S T_{k}(t)$ and $D U_{k}(t)$ are produced.

$$
\left[\mu_{\tilde{S}_{i}}\right] \text { and }\left[\mu_{\tilde{D}_{j}}\right] \text { for } i=1, \ldots m ; j=1, \ldots n
$$

Fuzzy values collected for a sensor are combined to form a matrix of membership values. For example all events related to the occupancy sensor are fuzzified and two matrices of fuzzy values for start time and duration are generated. This is summarised in the following expression:

$$
\begin{aligned}
\Sigma_{k}= & {\left[\begin{array}{ccccc}
\mu_{\tilde{S}_{1}^{1}} & \cdots & \mu_{\tilde{S}_{i}^{1}} & \cdots & \mu_{\tilde{S}_{m}^{1}} \\
\mu_{\tilde{S}_{1}^{2}} & \cdots & \mu_{\tilde{S}_{i}^{2}} & \cdots & \mu_{\tilde{S}_{m}^{2}} \\
\cdots & \cdots & \cdots & \cdots & \cdots \\
\mu_{\tilde{S}_{1}^{p}} & \cdots & \mu_{\tilde{S}_{i}^{p}} & \cdots & \mu_{\tilde{S}_{m}^{p}}^{p}
\end{array}\right]_{k} } \\
\Delta_{k}= & {\left[\begin{array}{ccccc}
\mu_{\tilde{D}_{1}^{1}} & \cdots & \mu_{\tilde{D}_{j}^{1}} & \cdots & \mu_{\tilde{D}_{n}^{1}} \\
\mu_{\tilde{D}_{1}^{2}} & \cdots & \mu_{\tilde{D}_{j}^{2}} & \cdots & \mu_{\tilde{D}_{n}^{2}} \\
\cdots & \cdots & \ldots & \ldots & \ldots \\
\mu_{\tilde{D}_{1}^{p}} & \cdots & \mu_{\tilde{D}_{j}^{p}} & \cdots & \mu_{\tilde{D}_{n}^{p}}
\end{array}\right]_{k} }
\end{aligned}
$$

where $p$ the total number of occurrences for a specific sensor, $k$. Each row of the matrix $\Sigma_{p \times m}$ and $\Delta_{p \times n}$ are membership 


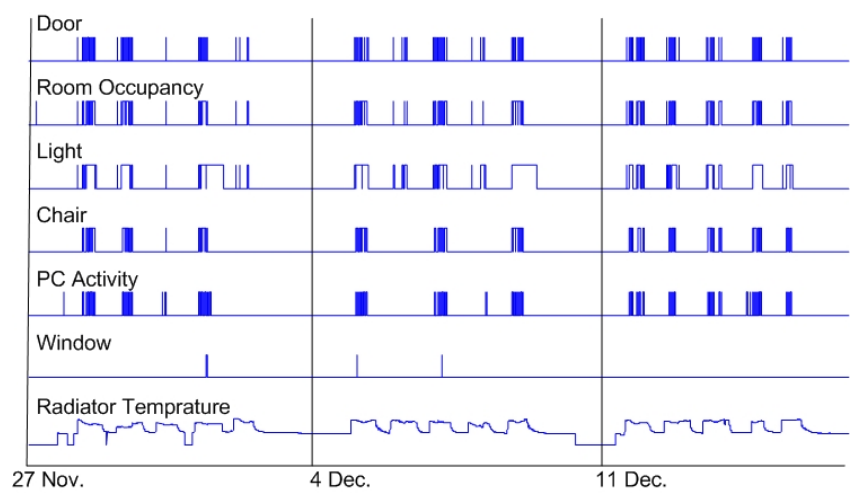

Fig. 5: A sample of a user activities over three weeks.

values for start time and duration of and event respectively. Number of rows are very much depends on the activity and the user profile.

To create a user profile based on each activity, we recommend creating a fuzzy characteristics matrix, $\Phi$ :

$$
\Phi_{k}=\Sigma_{k}^{T} \times \Delta_{k}
$$

$\Phi_{k}$ is a $n \times m$ matrix and this can be generated for all events and activities.

\section{EXPERIMENTS AND DISCUSSION}

The trial system was installed in three university offices occupied by workers with different patterns of work. For example, Fig. 5 shows a sample of the activities for a user over three weeks for our trial sensor networks. This data may be expanded as shown in Fig. 6 for the PIR and chair sensors for a Wednesday and Friday. It is extremely difficult to draw any conclusions about the occupant's behaviour from this form of representation.

In order to better represent the worker profile discussed earlier, applicable rules are identified for each day of the week. Since the data follows a weekly pattern, it is essential to take account of that aspect of the worker behaviour. Table III shows a comparison between two different office workers and the rules associated with their patterns. This allows us to simply categorise their power usage pattern for DPUS.

To illustrate the concept of fuzzy characteristics matrix discussed earlier, consider the room occupancy of a user over two separate days. The occupancy sensor has been activated/deactivated many time during the working day. Fuzzy characteristics matrices are created for both days of the data shown in Fig. 6. To visualise these profile matrices, they are displayed using scatter plots. Fig. 7 shows the worker

TABLE III: Typical worker profile for aggregated data.

\begin{tabular}{cccccc} 
& Mon. & Tue. & Wed. & Thur. & Fri. \\
\hline$\# 1$ & 42 & 17 & 42 & 5 & 17 \\
\hline$\# 2$ & 63 & 10 & 12 & 63 & 15 \\
\hline
\end{tabular}

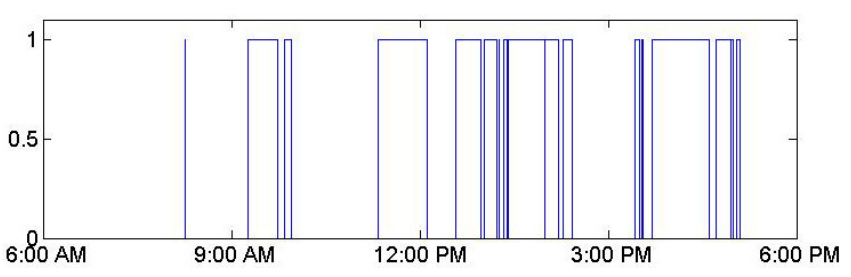

(a)

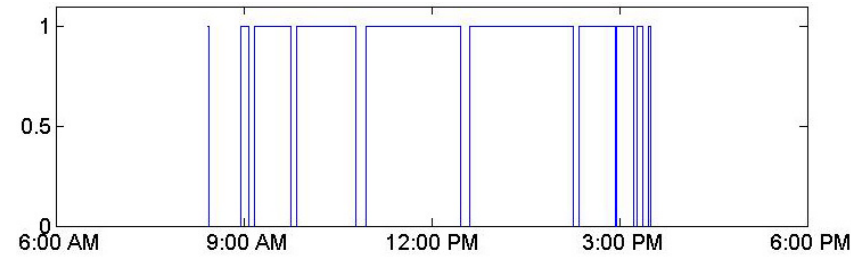

(b)

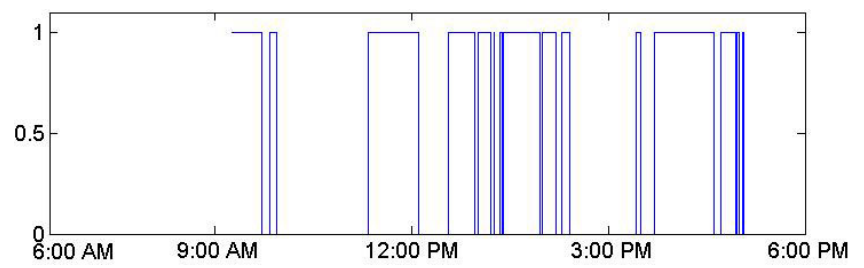

(c)

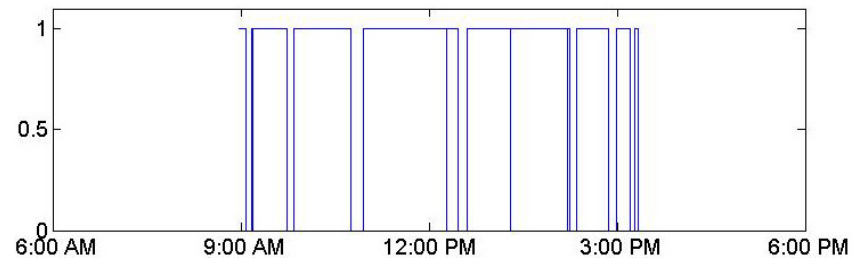

(d)

Fig. 6: Sensor activities (a) PIR activities for Wednesday (b) PIR activities for Friday (c) Chair activities for Wednesday (d) Chair activities for Friday .

behaviour emphasising the dominant patterns and clearly differentiates between two very different patterns of use.

In these plots, the size of the circle represents the likelihood of that activity occurrence for the specified start time and duration for that day of the week.

A prototype DPUS was constructed and significant energy savings were achieved without compromising the comfort and convenience of the worker, because the control was based on this interpretation of the typical worker characteristics. The experiment showed that the DPUS system used 33\% less energy than the current power management scheme. The calculation is mainly based on the power usage for PC.

\section{CONCLUSIONS AND FUTURE WORK}

In this paper we have explored methods of describing the behaviour of an office user using fuzzy values. It appears that to effectively model and control the office environment does not require the exact characteristics of the worker. Simplistic profiling seems to summarise and discriminate individual 


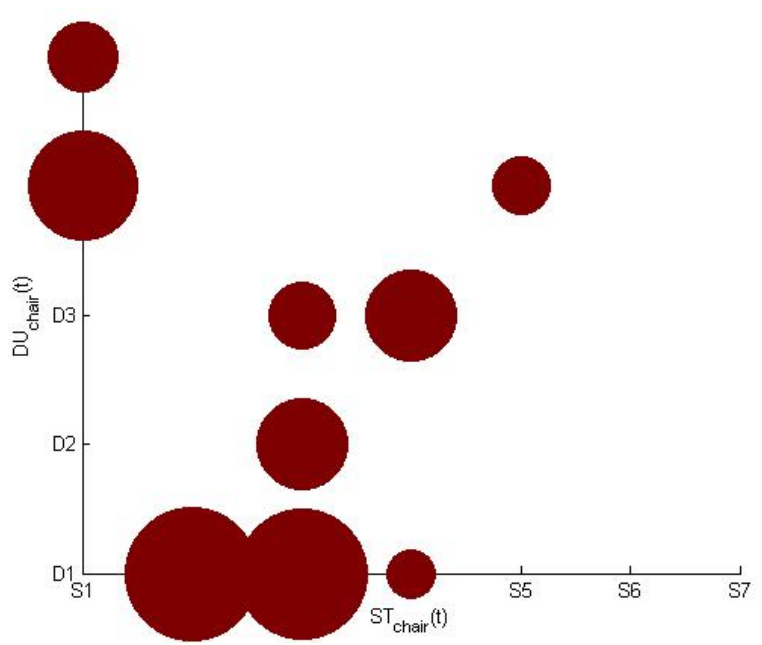

(a) Chair occupancy on Wednesday

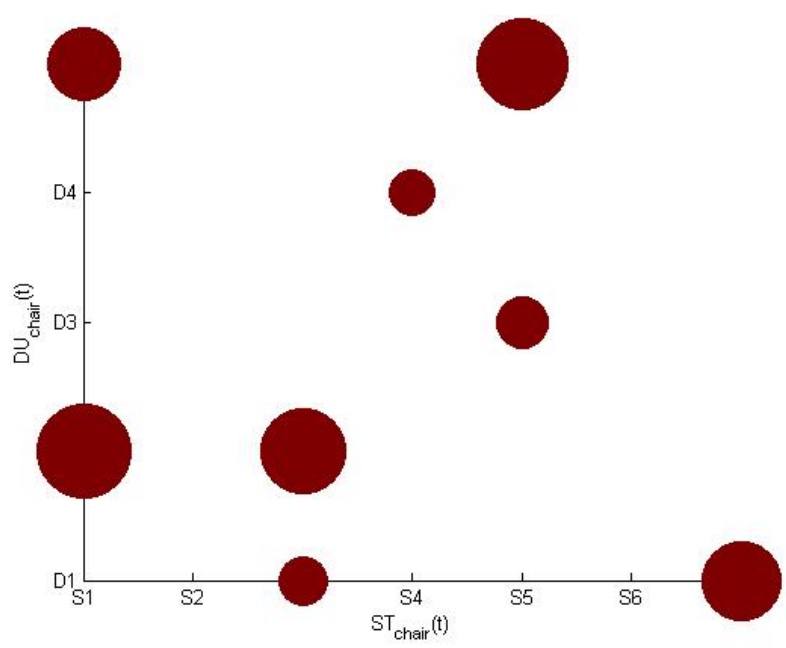

(b) Chair occupancy on Friday

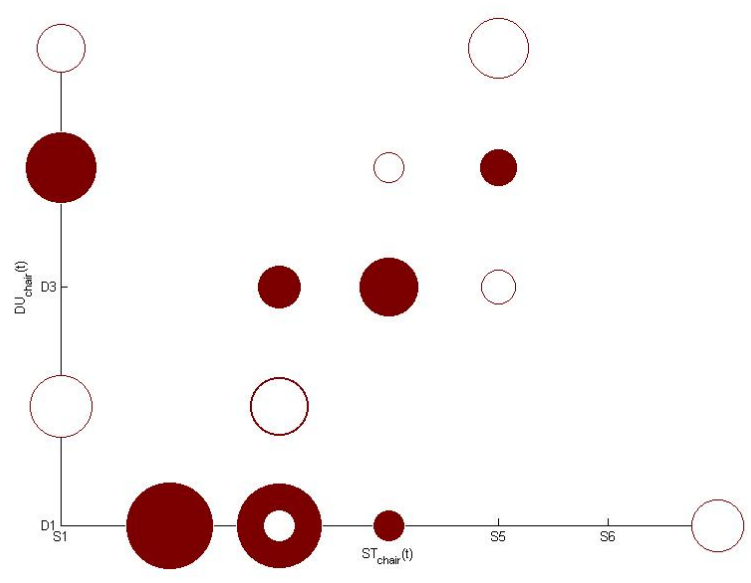

(c)

Fig. 7: Scatter plot for fuzzy characteristics matrix (a) Chair occupancy on Wednesday (b) Chair occupancy on Friday (c) Chair occupancy for both Wednesday and Friday. office workers effectively. A significant amount of energy was saved when using the prototype dynamic power management scheme compared with the university's power management scheme. For one PC over a typical day its energy usage was reduced by a third, with a saving of $0.1 \mathrm{kWh}$.

More data is required to continue the work investigating the seasonal variation of patterns in order to improve the energy management and prediction of activities. Identification of anomalous behaviour would also be beneficial in order to identify significant changes in office power use for example annual leave etc.

\section{REFERENCES}

[1] Jori Reijulaa, Matti Grohn, Kiti Muller, Kari Reijula, "Human wellbeing and flowing work in an intelligent work environment" Intelligent Buildings International, vol. 3, no. 4, 2011.

[2] L. W. Yen, Y. C. Wang, Y. C. Tseng, "iPower: an energy conservation system for intelligent buildings by wireless sensor networks" International Journal of Sensor Networks, vol. 5, no. 1, pp. 1-10, 2009.

[3] Bing Dong and Burton Andrews, "Sensor-Based Occupancy Behavioural Pattern Recognition for Energy and Comfort Management in Intelligent Buildings", Eleventh Int. IBPSA Conference, Glasgow, UK, July 27-30, 2009, pp. 1444-1451.

[4] Aries, M. B. C., Veitch, J. A., Newsham, G. R., "Physical and psychological discomfort in the office environment", Light, Performance and Quality of Life - Light and Health Research Foundation (SOLG) Symposium, 2007.

[5] Haynes, B., "The impact of office comfort on productivity", Journal of Facilities Management, Vol. 6 Iss: 1, pp.37-51, 2008.

[6] B. S. Lee, T. P. Martin, N. P. Clarke, B. Majeed, D. Nauck, "Dynamic daily-living patterns and association analyses in tele-care systems", in Proc. of the Fourth IEEE International Conference on Data Mining, Nov. 2004, pp. 447-450.

[7] Trevor Martin, Basim Majeed, Beum-Seuk Lee, Nick Clarke, "Fuzzy Ambient Intelligence for Next Generation Telecase", in Proc. of the IEEE Int. Conmf. on Fuzzy Systems, Vancouver, Canada, July 2006, pp. 894-901.

[8] Antti-Matti Vainio, Miika Valtonen, Jukka Vanhala, "Proactive Fuzzy Control and Adaptation Methods for Smart Homes", IEEE Intelligent Systems, Vol. 23, No. 2, pp. 42-49, 2008.

[9] Hani Hagras, Victor Callaghan, Martin Colley, Graham Clarke, Anthony Pounds-Cornish, Hakan Duman, "Creating an ambient-intelligence environment using embedded agents", IEEE Intelligent Systems, Vol. 9, No. 6, pp. 12-20, 2004

[10] Domenico Furno, Vincenzo Loia, Mario Veniero, Marco Anisetti, Valerio Bellandi, Paolo Ceravolo, Ernesto Damiani, "Towards an agent-based architecture for managing uncertainty in situation awareness", in Proc. of the 2011 IEEE Symposium on Intelligent Agent, April 2011.

[11] Saifullizam Puteh, Caroline Langensiepen, Ahmad Lotfi, "Similarity Pattern Mining in Intelligent Office Environments", in Workshop Proceedings of the 7th International Conference on Intelligent Environments, Vol. 10, 2011, pp. 562-573.

[12] Ahmad Lotfi, Caroline Langensiepen, Sawsan Mahmoud, MJ Akhlaghinia, "Smart Homes for the Elderly Dementia Sufferers: Identification and Prediction of Abnormal Behaviour", Journal of Ambient Intelligence and Humanized Computing, 2011. 